\title{
Videolaryngoscopy With Noninvasive Ventilation in Subjects With Upper-Airway Obstruction
}

\author{
Javier Sayas Catalán MD, Ignacio Jiménez Huerta MD, Pedro Benavides Mañas MD, \\ Manel Luján MD, Daniel López-Padilla MD, Eva Arias Arias MD, Ana Hernández Voth MD, \\ and Claudio Rabec MD
}

BACKGROUND: Noninvasive ventilation (NIV) titration may be difficult when dynamic airway obstruction episodes persist, even with high expiratory positive airway pressure (EPAP). We aimed to determine the usefulness of videolaryngoscopy during NIV for identifying mechanisms and sites of obstruction and for providing a guide for their resolution in difficult-to-titrate subjects. METHODS: When obstructions during NIV were present in the built-in software, EPAP was raised to $12 \mathrm{~cm} \mathrm{H}_{2} \mathrm{O}$. If obstructions persisted, a polygraphy during NIV was performed; if the events occurred with effort, a videolaryngoscopy with nasal and oronasal masks in awake subjects was performed. RESULTS: In a population of 208 subjects in whom NIV was initiated, 13 were identified as difficult to titrate with persistent obstructions during NIV despite an EPAP of $12 \mathrm{~cm} \mathrm{H}_{2} \mathrm{O}$. Videolaryngoscopy during NIV was able to identify the mechanism and the site of obstruction in all cases. The obstruction under oronasal mask ventilation was due to soft-palate (velum) collapse in 4 subjects, to epiglottic backward movement in 5 other subjects, and to tongue-base obstruction reducing the retroglossal space in 3 more. Videolaryngoscopy during NIV demonstrated improvement in 9 subjects $(69 \%)$ upon changing to nasal mask and suggested a possible surgical approach in $2(15 \%)$; in one of these 2 subjects, a successful uvulopalatopharyngoplasty was performed. CONCLUSIONS: The use of videolaryngoscopy during NIV in difficult-to-titrate patients may help to identify the sites and mechanisms of obstruction and in some cases may improve quality of ventilation. Key words: noninvasive ventilation; laryngoscopy; respiratory insufficiency; polysomnography; bi-level CPAP. [Respir Care 2017;62(2):222-230. (C) 2017 Daedalus Enterprises]

\section{Introduction}

The use of noninvasive ventilation (NIV) has increased notably over the last 2 decades in a variety of clinical

\footnotetext{
Drs Sayas Catalán, Benavides Mañas, López-Padilla, Arias Arias, and Hernández Voth are affiliated with the Servicio de Neumología, Hospital Universitario 12 de Octubre, Madrid, Spain. Dr López-Padilla is also affiliated with the Fundación para la Investigación Biomédica del Hospital General Universitario Gregorio Marañón, Madrid, Spain. Dr Jiménez Huerta is affiliated with the Otorhinolaryngology Department, Servicio de Neumología, Hospital Universitario 12 de Octubre, Madrid, Spain. Dr Luján is affiliated with the Servei de Pneumologia, Corporaciò Sanitària Parc Taulí, Hospital de Sabadell, Universitat Autònoma de Barcelona, Parc Taulí, Sabadell and CIBERES, Instituto de Salud Carlos III, Madrid, Spain. Dr Rabec is affiliated with the Service de Pneumologie et Soins Intensifs Respiratoire, Centre Hospitalier Universitaire de Dijon, Dijon, France.
}

conditions and scenarios. ${ }^{1}$ During NIV, a ventilator assists the patient, and events can be produced by the patient, by the ventilator or reflect a poor patient-ventilator synchrony. Attempts have been made to create a taxonomy and clas-

\footnotetext{
Drs López-Padilla and Sayas Catalán presented a version of this work at the American Thoracic Society 2015 International Conference, held May 15-20, 2015, in Denver, Colorado and at the $48^{\circ}$ Congreso Nacional SEPAR, held June 5-8, 2015, in Gran Canaria.

Dr Sayas Catalán has disclosed relationships with ResMed, Breas, PhilipsRespironics, Praxair-Oximesa, and Covidien. The other authors have disclosed no conflicts of interest.

Correspondence: Javier Sayas Catalán MD, Servicio de Neumología, Hospital Universitario 12 de Octubre, Avda de Córdoba, s/n, 28041 Madrid, Spain. E-mail: jsayascat@gmail.com.
}

DOI: $10.4187 /$ respcare. 04784 
sification of the different events found in respiratory polygraphy or polysomnography under NIV.,2,3 Intermittent obstruction of the upper airway is a common phenomenon and may be related to 2 mechanisms. The first corresponds to oropharyngeal obstructive events due to upper-airway collapse, as a result of insufficient expiratory positive airway pressure (EPAP). This mechanism may be present in patients with unstable upper airway, such as those with obstructive sleep apnea or obesity-hypoventilation syndrome. These events are characterized by increased inspiratory activity, indicating a struggle against upper-airway collapse. Current recommendations indicate how to adjust the level of pressure to solve those obstructive events. ${ }^{4}$ The second mechanism corresponds to intermittent glottis obstruction induced by cyclic hypocapnia, where the main feature is a decrease in or an abolition of inspiratory effort. Positive-pressure ventilation-induced hyperventilation has been shown to promote active glottis closures in normal subjects when either awake or asleep. ${ }^{5,6}$ In this case, the suggested approach is to reduce minute ventilation. ${ }^{3,4}$

When monitoring a patient during NIV using an overnight $\mathrm{S}_{\mathrm{pO}_{2}}$ recording, even if coupled to the built-in software of the ventilator, ${ }^{7}$ these 2 mechanisms are indistinctly expressed as desaturation dips. A ventilator polygraphy or polysomnography recording is needed to distinguish between the 2 mechanisms according to the effort pattern. ${ }^{3}$

Nasal masks and facial masks are both used to provide NIV. A review of published studies showed that during acute NIV, facial masks predominate (63\%) but that nasal masks are most commonly used for chronic NIV. ${ }^{8}$ It has been suggested that, in patients with obstructive sleep apnea, a facial mask is tolerated worse than a nasal mask ${ }^{9}$ and that adherence is higher with nasal masks. ${ }^{10}$ Recent data have suggested that the residual apnea-hypopnea index (AHI) is significantly lower in subjects treated with nasal masks and that higher pressures are needed when a facial mask is used. ${ }^{11}$ Additionally, 2 reports described cases in which CPAP and NIV were not effective when a facial mask was used despite a progressive rise in expiratory pressure, whereas treatment with nasal NIV with lower pressures was adequate. ${ }^{12,13}$ The current study describes a cohort of subjects, all wearing facial masks and exhibiting persistent obstructive events that were refractory to the increasing pressure titration performed according to current guidelines. ${ }^{4}$ These subjects were assessed by dynamic endoscopy as part of the usual clinical workup to evaluate the upper airway in spontaneous breathing and under NIV with different pressure levels. The aim of the study was to describe the different locations and patterns of upper-airway obstruction in difficult-to-titrate subjects and to analyze the impact of the endoscopic findings on the NIV titration process.

\section{QUICK LOOK}

\section{Current knowledge}

NIV titration is performed by using either polygraphy or polysomnography both to preserve upper airway patency and to support ventilation. If residual obstructive events with increased inspiratory effort remain, the current recommendation is to progressively raise expiratory pressure to stabilize the upper airway. However, in some cases, obstructive events persist despite the use of high expiratory pressures. In these difficult-to-titrate patients the specific mechanisms explaining upper-airway obstruction events remain unclear.

\section{What this paper contributes to our knowledge}

In more than half of difficult-to-titrate subjects, fiberoptic videolaryngoscopy under NIV helped to identify anatomic and functional mechanisms underlying upperairway obstruction. Hence, a systematic evaluation using this technique guided physicians to choose the most appropriate strategy to improve the quality of ventilation.

\section{Methods}

\section{Setting}

The Hospital Universitario 12 de Octubre in Madrid, Spain, is a 1,300-bed teaching hospital that is a reference center for long-term mechanical ventilation. It cares for patients with obesity-hypoventilation syndrome, COPD, and neuromuscular disorders.

\section{Subjects and Ethics}

Subjects with indication for long-term NIV, consecutively recruited between September 2012 and December 2014, were included in the study. Long-term NIV was considered in 2 situations: (1) subjects with chronic respiratory failure in whom elective NIV was indicated according to the recommendations of the 1999 American College of Chest Physicians and consensus ${ }^{14}$ and (2) subjects recovering from an acute-on-chronic respiratory failure event in whom the indication for long-term NIV was maintained. In the latter case, the evaluation was performed at least 1 month after discharge so as to ensure clinical stability. Thus, in both situations, all subjects had been in a stable clinical condition for at least 1 month at inclusion. This observational study was approved by the institutional review board of the Hospital Universitario 12 de Octubre (CEIC 084), which considered that the study corresponds to routine care. 


\section{Usual NIV Titration Protocol}

Adaptation to long-term NIV was performed in hospital, under the surveillance of a senior pulmonologist and trained nurses. NIV was commenced in accordance with published guidelines. ${ }^{4}$ All subjects were initiated on NIV with pressure support ventilation with a backup breathing frequency. The backup rate was calculated as -2 breaths/min below the spontaneous awake breathing frequency. The efficacy of ventilation was assessed by performing overnight oximetry coupled to transcutaneous capnography (V-Sign, SenTec, Therwil, Switzerland) and/or early morning arterial blood gases. Trends and raw data were obtained from built-in software coupled to the ventilator. Three different built-in monitoring systems were used: ResCAN (for the Stellar 150 and VPAP S9 ventilators) (ResMed, Sydney, Australia), Direct View (for the Trilogy ventilator) (Philips Respironics, Murrysville, Pennsylvania), and Vivo software (for the VIVO 50 ventilator) (Breas Medical AB, Mölnlycke, Sweden). Settings were gradually adjusted according to the monitoring results to achieve the outcomes of satisfactory titration reported by the SomnoNIV group. ${ }^{3,7}$ If this simplified monitoring showed significant cumulated desaturation dips ( $>3 \%$ for $>10 \%$ of the recording) and/or the built-in software displayed relevant flow-limitation events ( $>30 \%$ of decrease for $>10 \%$ of recording) although EPAP was raised to $12 \mathrm{~cm} \mathrm{H}_{2} \mathrm{O}$, subjects were considered as presenting persistent obstructive events refractory to increasing pressure titration, and an overnight ventilatory polygraphy was scheduled. To avoid the "first night effect" and to ensure that the subject was well adapted to NIV, a minimum adaptation time of 4 weeks between the beginning of the adaptation and the polygraphy was required.

Ventilatory polygraphy was performed using an Embletta Gold polygraph (Embla Systems, Denver, Colorado). This polygraphy included, at least, pneumotachographbased air flow, mask pressure, thoraco-abdominal movements, and $\mathrm{S}_{\mathrm{pO}_{2}}$ recordings. The pneumotachograph was placed between the circuit and the mask (with an intentional leak). The respiratory events were scored manually in accordance with the SomnoNIV recommendations. ${ }^{3}$ Briefly, a respiratory event was defined as a modification, discontinuity, or instability of ventilation for $\geq 10 \mathrm{~s}$, with deleterious consequences for $\mathrm{S}_{\mathrm{pO}_{2}}, \mathrm{P}_{\mathrm{tcCO}_{2}}$, and/or sleep. Upper-airway obstruction events were defined by a decrease in flow, while the amplitude of pressure signal remained unchanged, and were scored as upper-airway obstruction with increasing ventilatory drive when the flow reduction had a simultaneous progressive increase of abdominal and thoracic belt signals, with or without a phase opposition. An upper-airway obstruction with decreasing ventilatory drive was considered when the decrease in flow had a simultaneous reduction or disappearance of thoracic

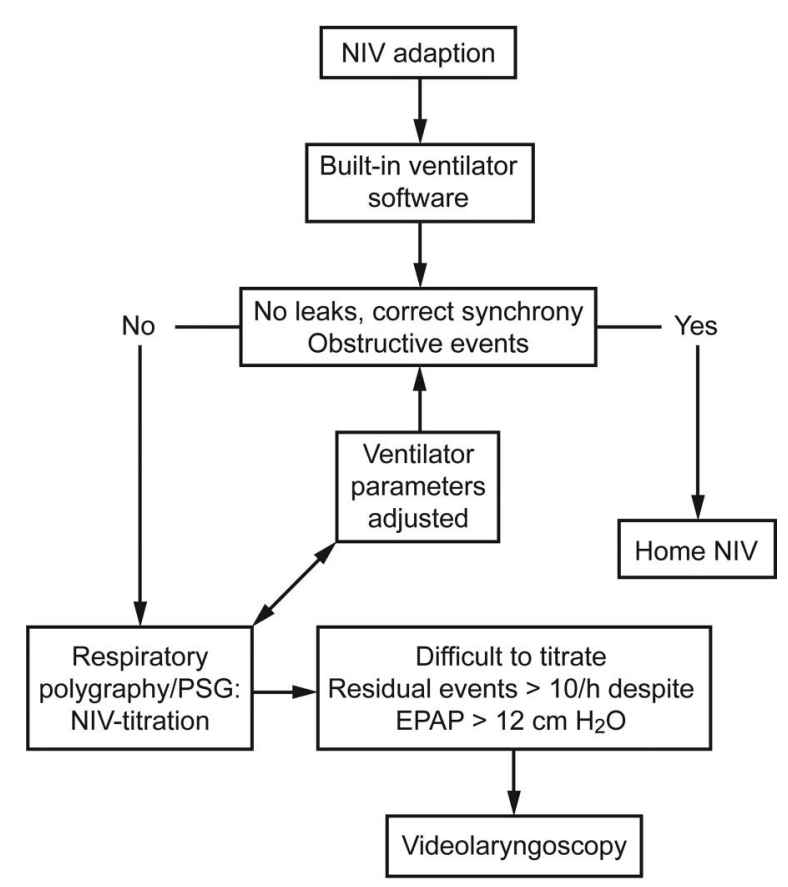

Fig. 1. Protocol flow chart.

and abdominal belt signal without phase opposition. Figure 1 summarizes the study protocol.

\section{Videolaryngoscopy Under the Noninvasive Ventilation Protocol}

The same ear-nose-throat surgeon (IJH) performed all the videolaryngoscopies. The upper airway was examined using a fiber-optic nasopharyngoscope (4.3-mm diameter) (Machida, Chiba, Japan). The device was coupled to a video system to record all of the fiber-optic evaluations and to allow subsequent review and evaluation by another independent specialist. The examination was performed with the subject awake, in supine position (head resting at $30^{\circ}$ ). To avoid changes in airway patency, topical anesthesia and vasoconstrictors were avoided. A first evaluation was made during spontaneous breathing, and the entire upper aero-digestive tract was exhaustively inspected and recorded. Afterward, a second evaluation was performed under NIV. Videolaryngoscopy under NIV was carried out via a facial mask designed for endoscopy (Intersurgical Explorer, Intersurgical, Wokingham, United Kingdom). Finally, a third evaluation was performed using a newborn-size mask (Intersurgical Explorer) as a nasal mask. In each case, during endoscopy, ventilation was performed by setting inspiratory and expiratory pressures close to those previously used in the adaptation. They were then gradually increased up to $12 \mathrm{~cm} \mathrm{H}_{2} \mathrm{O}$ of EPAP and up to a maximum of $24-26 \mathrm{~cm} \mathrm{H}_{2} \mathrm{O}$ of inspiratory 


\section{VIDEOLARYNGOSCOPY WITH NIV}

positive airway pressure (IPAP), until the airway obstruction was corrected; if the obstructions persisted beyond these limits, the pressures were not increased further. Subsequent therapeutic modifications were proposed after videolaryngoscopy findings, and a further polygraphy was performed to check their effectiveness.

$\mathrm{P}_{\mathrm{aCO}}$, levels and AHI were documented at baseline $\left(\mathrm{P}_{\mathrm{aCO}}{ }^{-1}\right.$ and $\left.\mathrm{AHI}-1\right)$, after the first polygraphy $\left(\mathrm{P}_{\mathrm{aCO}}{ }^{-2}\right.$ and AHI-2), and after videolaryngoscopy therapeutic adjustments with a second polygraphy $\left(\mathrm{P}_{\mathrm{aCO}_{2}}{ }^{-3}\right.$ and AHI-3).

\section{Statistics}

Quantitative values are presented as means \pm SD or medians with interquartile range. Pre- and post-AHI and $\mathrm{P}_{\mathrm{aCO}_{2}}$ values were compared using the paired Student $t$ test or, in the case of non-normal distributions, the Wilcoxon signed-rank test. Significance was set at $P<.05$. Statistical analysis was performed using SPSS 21.0 for Windows (SPSS, Chicago, Illinois).

\section{Results}

During the study period, home NIV was initiated de novo in 208 subjects. Obesity-hypoventilation syndrome (52\%) was the main etiology leading to NIV indication. Mean age was $67.2 \pm 12.5 \mathrm{y}$, and $112(54 \%)$ were females. Difficult titration was observed in 13 subjects (6\%), who presented a mean age of $71.4 \pm 16.9 \mathrm{y}$ and mean body mass index of $30.6 \pm 9.4 \mathrm{~kg} / \mathrm{m}^{2} ; 7(54 \%)$ were females. The mean backup rate was $13.5 \pm 1.2$ breaths/min. After reaching clinical stabilization, baseline $\mathrm{pH}$ was $7.40 \pm 0.44$, baseline $\mathrm{P}_{\mathrm{aCO}_{2}}$ was $68.6 \pm 11.2 \mathrm{~mm} \mathrm{Hg}$, and baseline AHI (AHI-1) was $44.5 \pm 22.2$ events/h. COPD, obesity-hypoventilation syndrome, and kyphoscoliosis were the most frequent diagnoses included. Table 1 summarizes baseline characteristics, including sleep study features. Once the ventilatory polygraphy was performed, the mean obstructive events index per hour (AHI-2) was $37.5 \pm 21.4$ events/h. Videolaryngoscopy findings are presented in Table 2, and Figures 2 and 3 present features related to the procedure.

After the videolaryngoscopy findings, individualized treatment was proposed in each clinical scenario. In 6 subjects (46\%), a nasal mask was introduced along with lower IPAP and EPAP levels and supplementary oxygen, whereas 3 subjects (23\%) continued with a facial mask, although both IPAP and EPAP were reduced due to substantial air leakage. CPAP therapy was proposed for 3 subjects (23\%) who declined NIV. One of these 3 subjects also underwent an uvulopalatopharyngoplasty and presented a noticeable improvement. Finally, one kyphoscoliotic subject $(15.4 \%)$ with a curly epiglottis and entire hypopharynx obstruction under NIV did not improve after
Table 1. Baseline Characteristics, Gasometrical Values, and Sleep Study Features

\begin{tabular}{lc}
\hline \hline \multicolumn{1}{c}{ Characteristics } & Values \\
\hline Female, $n(\%)$ & $7(54)$ \\
Age, mean $\pm \mathrm{SD} \mathrm{y}$ & $71.4 \pm 16.9$ \\
BMI, mean $\pm \mathrm{SD} \mathrm{kg} / \mathrm{m}^{2}$ & $30.6 \pm 9.4$ \\
Diagnosis, $n(\%)$ & \\
OHS & $4(31)$ \\
Overlap syndrome (COPD + OHS) & $3(23)$ \\
Kyphoscoliosis & $3(23)$ \\
Amyotrophic lateral sclerosis & $1(7.7)$ \\
Mitochondrial myopathy & $1(7.7)$ \\
COPD & $1(7.7)$ \\
Mallampati score, $n(\%)$ & $1(7.7)$ \\
I & $3(23)$ \\
II & $6(46)$ \\
III & $3(23)$ \\
IV & $67.7 \pm 11.2$ \\
$\mathrm{P}_{\text {aCO }}$, mean \pm SD mm Hg & $44.5 \pm 22.2$ \\
AHI-1, mean \pm SD & \\
& \\
\hline BMI $=$ body mass index & \\
OHS $=$ obesity-hypoventilation syndrome & \\
AHI $=$ apnea-hypopnea index & \\
\hline
\end{tabular}

Table 2. Videolaryngoscopy Findings After Ventilatory Polygraphy

\begin{tabular}{ll}
\hline \hline \multicolumn{1}{c}{ Findings } & Values \\
\hline VL in spontaneous ventilation & \\
Redundant soft-palate tissue and uvular elongation & $4(31)$ \\
Normal upper airway & $4(31)$ \\
Reduced retroglossal space & $3(23)$ \\
Altered epiglottis shape & $3(23)$ \\
$\quad$ Curly & $2(15.4)$ \\
$\quad$ Omega-shaped & $1(7.7)$ \\
VL under noninvasive ventilation with an oronasal interface & \\
Epiglottis shifted backwards (see Figs. 2 and 3) & $5(38)$ \\
Soft-palate collapse & $4(31)$ \\
Retroglossal collapse & $3(23)$ \\
Paradoxical vocal fold movements & $2(15.4)$ \\
VL under noninvasive ventilation with a nasal interface & \\
$\quad$ No relevant changes & $7(54)$ \\
Oropharynx patency improvement (see Fig. 4) & $3(23)$ \\
Epiglottis stability & $2(15.4)$ \\
Hypopharynx patency & $1(7.7)$ \\
& \\
\hline Results are $n$ (\%). & \\
VL = videolaryngoscopy & \\
\hline
\end{tabular}

attempting several treatments, including switching to a nasal interface and reducing NIV pressures. All subjects except for the one just mentioned improved with the proposed treatments. Tables 3 and 4 show statistically significant reductions in $\mathrm{P}_{\mathrm{aCO}}$ levels and in the residual obstructive event index after videolaryngoscopy. 

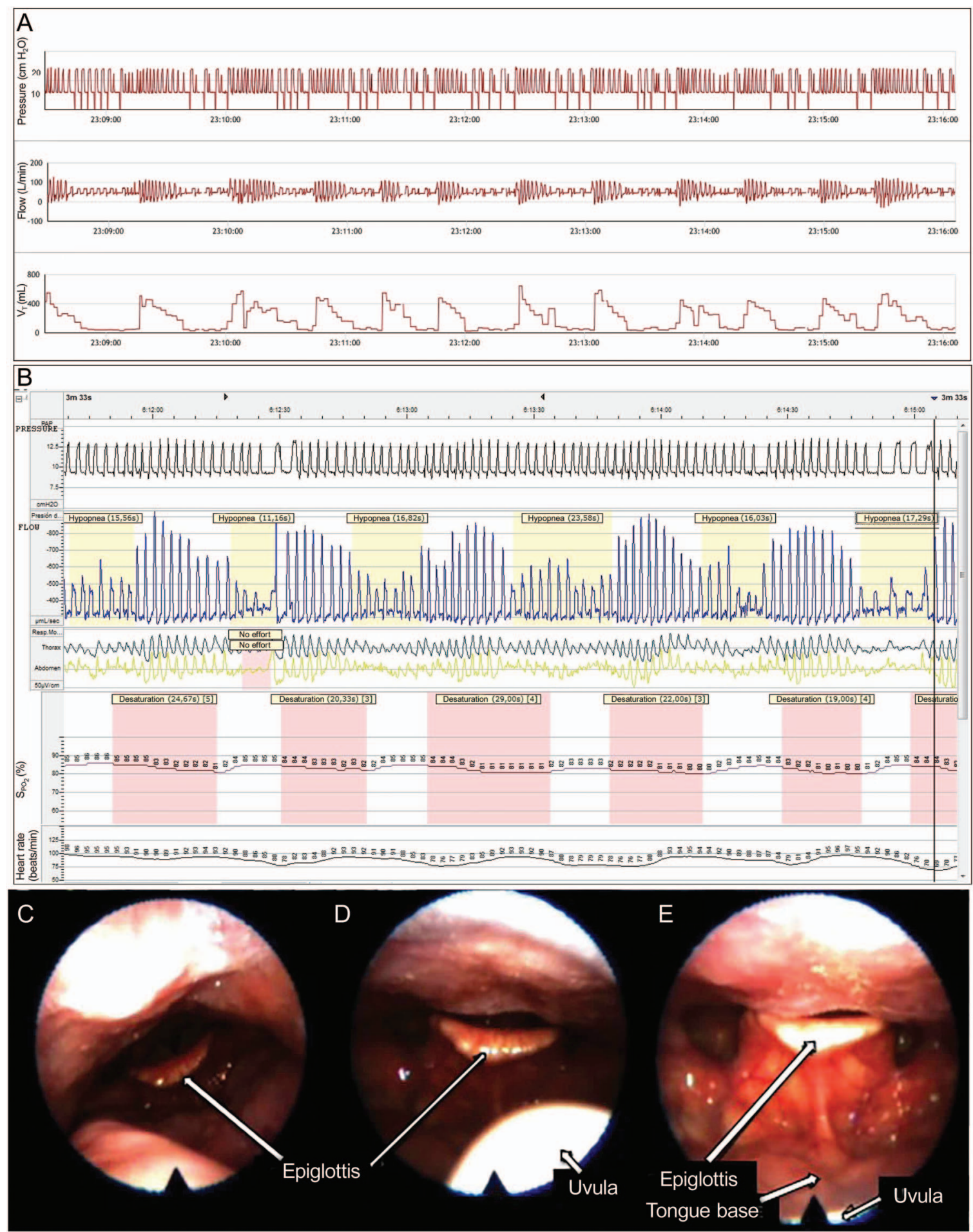

Fig. 2. Subject with ALS. Direct View (Philips Respironics) built-in software shows recurrent obstructive events under noninvasive ventilation (oronasal mask, inspiratory positive airway pressure [IPAP] 22, expiratory positive airway pressure [EPAP] 12) (A), ventilatory polygraph (pressure, flow, $\mathrm{S}_{\mathrm{pO}_{2}}$, respiratory movements) (B), and videolaryngoscopy findings (C-E). Note how the epiglottis is shifted backwards, aggravating the obstruction as the pressure increases. Images displayed correspond to spontaneous breathing (C), IPAP 10, EPAP 4 (D), and IPAP 18, EPAP 12 (E). 


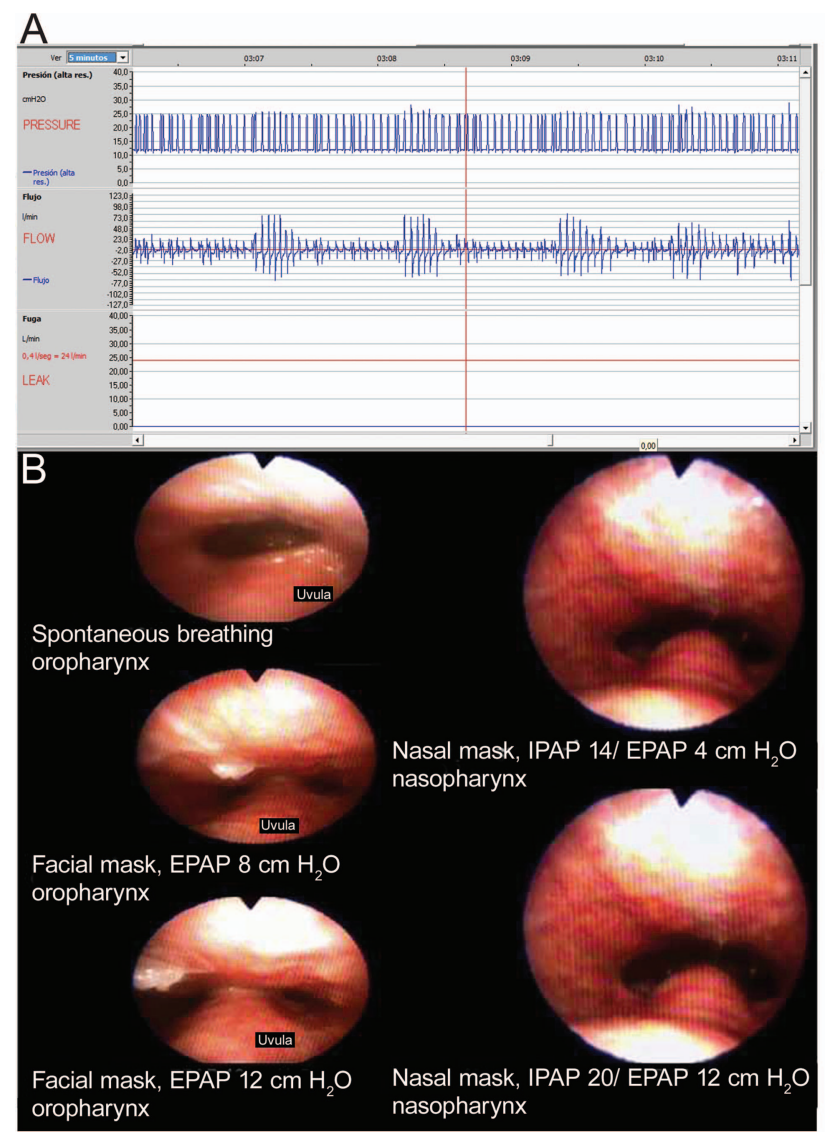

Fig. 3. ResCAN (ResMed) traces under noninvasive ventilation (facial mask, inspiratory positive airway pressure [IPAP] 24, expiratory positive airway pressure [EPAP] 12) (A) are displayed in a 5-min time lapse, showing recurrent upper-airway obstructions. Bottom: the oropharynx is obstructed as IPAP and EPAP increase by pushing the soft palate and uvula backwards. With a nasal mask, even at much lower pressures, the obstruction is resolved (B).

\section{Discussion}

The main findings of our study can be summarized as follows. First, videolaryngoscopy under NIV may help to identify the mechanism and site of upper-airway obstruction in difficult-to-titrate subjects. Second, endoscopic findings can help the clinician to choose alternative approaches during NIV (eg, modifying IPAP/EPAP values, changing the mask, or even proposing surgery).

To our knowledge, in subjects with an indication of home NIV, no systematic endoscopic evaluation of the upper-airway response to bi-level positive pressure has been reported, although the reaction of the glottis to hyperventilation in healthy volunteers was described as early as 1995 by Jounieaux et al.,5,15 Neuromuscular patients with bulbar impairment, particularly amyotrophic lateral sclerosis (ALS), are more likely to fail NIV than those without bulbar impairment. ${ }^{16,17}$ In a recent study evaluating 179 subjects with ALS, Georges et al ${ }^{18}$ found that 48
(27\%) remained inadequately ventilated after optimal adjustment of ventilator settings at night as a result of airway-obstructive events. Interestingly, the 1-y survival in those subjects was significantly lower than in their adequately ventilated peers. Epiglottic flapping due to bulbar muscle weakness may be another explanation for the failure of NIV therapy. Ito et al ${ }^{19}$ reported a case of floppy epiglottis in 2 subjects with ALS, which was only evaluated in spontaneous ventilation. Sanches et al ${ }^{20}$ reported another case, a subject with obstructive sleep apnea, in whom a floppy epiglottis was responsible for poor CPAP response. Moreover, a Swedish study of upper-airway changes in healthy volunteers using mechanical cough assist $^{21}$ found an epiglottic retroflexion occluding the whole laryngeal inlet in some cases. Similarly, the way NIV is applied (ie, on the basis of ventilator parameters, especially IPAP) can aggravate the epiglottic dysfunction. When high pressure values are used, the epiglottic dysfunction may result in the occlusion of the laryngeal spaces. These findings should be kept in mind as possible exceptions to the recommendations that both inspiratory and expiratory pressures should be increased in the presence of an upperairway obstruction. ${ }^{20}$

The role of the epiglottic anatomy in favoring these obstructive events is unknown. Almost all of our subjects with floppy epiglottis presented a curly-shaped anatomy. We also found obstructions in one subject with an omegashaped epiglottis, but little is known at present regarding the reactions of these anatomic variations to different levels of pressure. Previous studies assessing the endoscopic patterns of post-uvulopalatopharyngoplasty residual obstructions in a general population found the epiglottis to be a site of obstruction in $22 \%$ of subjects. ${ }^{22}$ As suggested, the possibility of a surgical approach such as a partial U-shaped epiglottectomy should be considered in these subjects when epiglottic abnormalities are found.

As explained before, upper-airway obstruction in patients receiving NIV could be located either at the oropharyngeal or at the glottic level. In a ventilatory polygraphy, both obstructive events will display a reduction in inspiratory flow. However, in the first case, this reduction is associated with a progressive increase in inspiratory effort, whereas in the second case, a reduction or absence of thoracic and belt signals is shown.

In our series, all subjects presented obstructive events with increased inspiratory effort. As expected, endoscopy indicated that the site of the obstruction in most subjects was the oropharynx or above the glottis (ie, soft-palate collapse or tongue-base obstruction; see Tables 2 and 5). However, in subjects 6 and 10, the obstruction was situated in the glottis. These findings challenge the classical definition of glottis closure and suggest the possibility of another mechanism, related to passive hyperventilation. Georges et al ${ }^{18}$ describe the same mechanism in an ALS 
Table 3. Comparison of $\mathrm{P}_{\mathrm{aCO}}$ Levels Before and After Videolaryngoscopy Under Noninvasive Ventilation

\begin{tabular}{lllr}
\hline \hline \multirow{2}{*}{ Sample } & \multicolumn{3}{c}{$\mathrm{P}_{\mathrm{aCO}_{2}(\mathrm{~mm} \mathrm{Hg})}$} \\
\cline { 2 - 3 } & Before VL-NIV & After VL-NIV \\
\hline Overall population $(n=13)$ & $72.0(60.5-78.0)$ & $53.8(49.5-58.0)$ & $<.001$ \\
Nasal interface $(n=6)$ & $73.0(61.2-83.8)$ & $54.8(49.0-59.8)$ & .004 \\
Lower bi-level pressures $(n=8)$ & $67.5(57.2-80.0$ & $54.5(47.8-58.5)$ & .002 \\
CPAP $(n=3)$ & $76.0(64.0-79.0)$ & $51.3(49.0-54.0)$ & .12
\end{tabular}

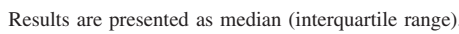

* Non-parametric test (Wilcoxon).

VL-NIV = videolaryngoscopy under noninvasive ventilation

Table 4. Comparison of Ventilatory Polygraphy Apnea-Hypopnea Index and Built-In Software Apnea-Hypopnea Index After Videolaryngoscopy Under Non-Invasive Ventilation

\begin{tabular}{llll}
\hline \hline \multicolumn{1}{c}{ Sample } & AHI-2 (events/h) & AHI-3 (events/h) & $P^{*}$ \\
\hline Overall population $(n=13)$ & $33.0(21.5-54.5)$ & $4.0(1.6-18.0)$ & .004 \\
Nasal interface $(n=6)$ & $26.5(17.0-32.2)$ & $1.8(0.8-6.7)$ & .10 \\
Lower bi-level pressures $(n=8)$ & $30.0(19.8-42.8)$ & $2.4(1.1-14.5)$ & .035 \\
CPAP $(n=3)$ & $58.0(34.0-76.0)$ & $7.0(5.0-18.0)$ & .11
\end{tabular}

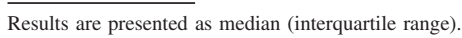

* Non-parametric test (Wilcoxon).

AHI-2 = ventilatory polygraphy apnea-hypopnea index

AHI-3 = built-in software apnea-hypopnea index

population receiving NIV. Therefore, in some patients, especially in those with bulbar dysfunction, the mechanism of glottis closure may be related not to positive pressure-induced hyperventilation but rather to intrinsic vocal cord dysfunction. Further studies are needed to examine these different mechanisms in more detail.

Another result is that $40 \%$ of subjects benefited from shifting the interface to a nasal mask. In some subjects, the use of a facial mask can aggravate obstructive events. The suggested mechanism for this obstruction is a retrognatia induced by the pressure of the mask at the chin, pushing the whole mandible backwards and narrowing the anterior-posterior airway diameter. ${ }^{13,23}$ When pressures are increased to overcome this tongue-base obstruction, a paradoxical obstruction due to a downward shift of the tongue base may take place.

Some authors have suggested that, in a subset of patients at least, the use of facial masks may be less effective than nasal masks, due to a similar mechanism. High pressure air pushes the tongue base backwards, collapsing the pharynx and flopping the epiglottis to obstruct the airway inlet. ${ }^{10,12,13,23,24}$ A randomized controlled trial studying a cohort of subjects with sleep apnea treated with CPAP showed a significantly lower residual AHI when subjects wore a nasal mask rather than a face mask. ${ }^{24}$ Similarly, a recent prospective observational cohort study suggested that the use of a facial mask was associated with higher CPAP pressure requirements than both nasal masks and nasal pillows. ${ }^{22}$ Our study may provide an explanation for these findings.

Several limitations of this study should be mentioned. First, we performed the videolaryngoscopy in awake subjects. Sleep-induced endoscopy has been proposed as a more effective procedure for identifying obstruction sites than endoscopy in awake subjects breathing spontaneously. ${ }^{25}$ However, because the sites and mechanism of obstruction were clearly identified in all cases, we did not consider sleep-induced endoscopy to be necessary, especially bearing in mind that it requires sedation and is not a risk-free procedure. Second, titration was performed according to the SomnoNIV recommendations, mainly with polygraphy. ${ }^{2,3}$ As a result, most subjects did not undergo full polysomnography as proposed by the American Academy of Sleep Medicine. ${ }^{4}$ In our environment, access to repeated polysomnography is limited. Polygraphy does not allow an assessment of sleep efficiency, and upper-airway obstruction (even mechanically induced) is believed to occur or be aggravated during sleep. As a result, it is possible that the severity (or the number of subjects with difficult titration) was underestimated in this study. In addition, the improvements after therapeutic modifications may have been due to night-by-night variability in sleep efficiency. Recently, an approach based on the analysis of built-in ventilator software has been proposed. ${ }^{7}$ In a small number of subjects, Georges et $\mathrm{al}^{26}$ found a high degree of corre- 


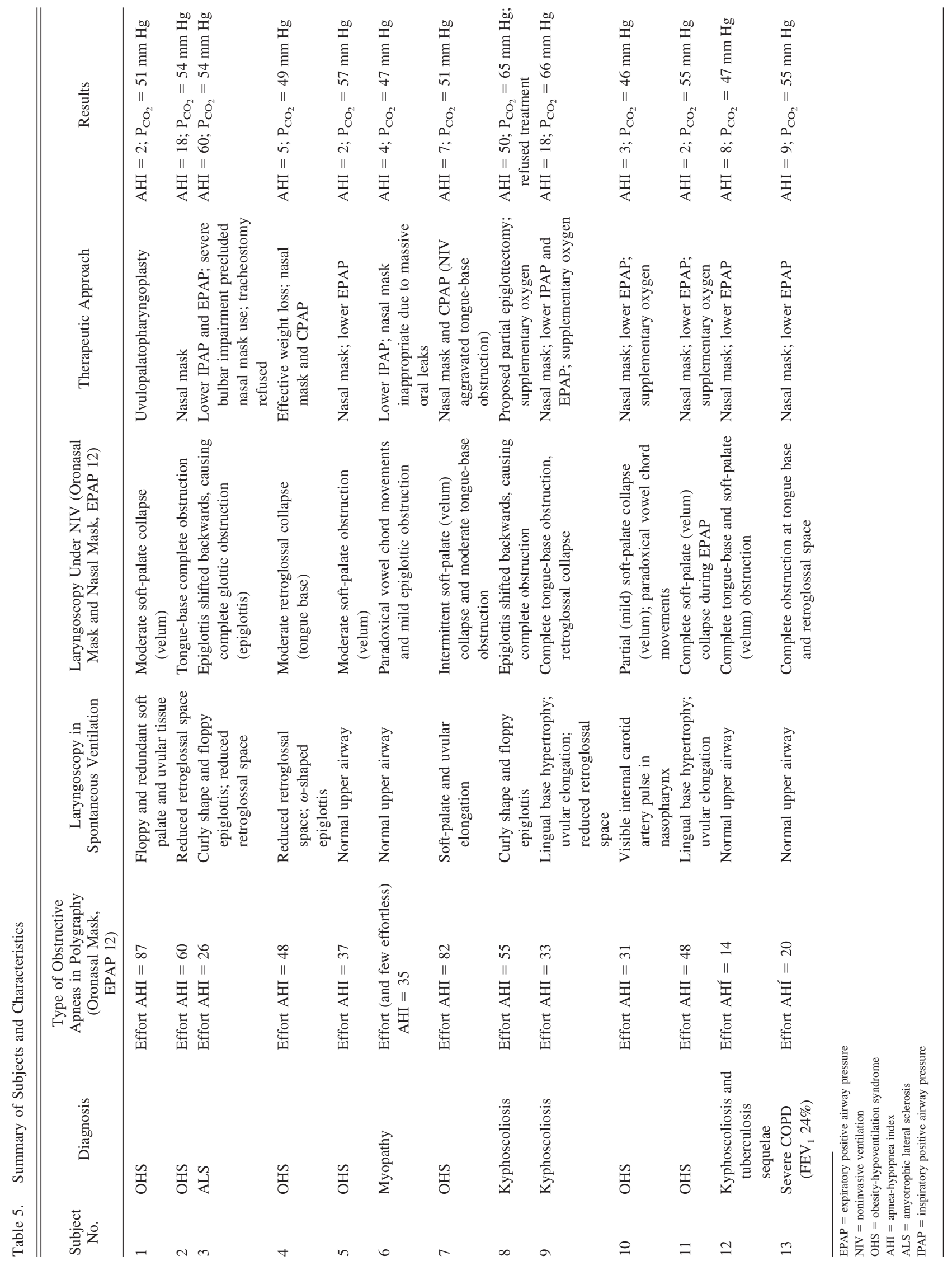




\section{VIDEOLARYNGOSCOPY WITH NIV}

lation of the automatic AHI obtained by commercial software with the AHI obtained by full polysomnography. Third, we found a variety of obstruction mechanisms and propose modifications based on our findings. However, we were unable to assess the impact of other therapeutic approaches; the addition of mandibular advance devices, for example, may improve airway obstruction in subjects with sleep apnea who are wearing facial masks. ${ }^{23}$ Finally, we have no data concerning $\mathrm{P}_{\mathrm{CO}_{2}}$ kinetics during endoscopic evaluations. Because $\mathrm{P}_{\mathrm{CO}_{2}}$ variations may have a significant impact on airway patency,5, 6,18 this may account for the differences observed when subjects were assessed in the 3 different situations (spontaneous breathing and ventilation with facial or nasal mask).

\section{Conclusions}

Subjects who were difficult to titrate due to upper-airway obstruction represented $6 \%$ of our series of candidates for home NIV. In these subjects, a systematic evaluation with fiberoptic videolaryngoscopy during spontaneous breathing and while receiving NIV could help to identify the site of obstruction. The use of this tool provides an anatomy-based approach with the potential to improve NIV effectiveness.

\section{ACKNOWLEDGMENTS}

We thank Mireia Carvajal Cornago for help with the English version of the manuscript.

\section{REFERENCES}

1. Carpe-Carpe B, Hernando-Arizaleta L, Ibanez-Perez MC, PalomarRodriguez JA, Esquinas-Rodriguez AM. Evolution of the use of noninvasive mechanical ventilation in chronic obstructive pulmonary disease in a Spanish region, 1997-2010. Arch Bronconeumol 2013;49(8):330-336.

2. Gonzalez-Bermejo J, Perrin C, Janssens JP, Pepin JL, Mroue G, Léger $\mathrm{P}$, et al. Proposal for a systematic analysis of polygraphy or polysomnography for identifying and scoring abnormal events occurring during non-invasive ventilation. Thorax 2012;67(6):546-552.

3. Rabec C, Rodenstein D, Leger P, Rouault S, Perrin C, GonzalezBermejo J. Ventilator modes and settings during non-invasive ventilation: effects on respiratory events and implications for their identification. Thorax 2011;66(2):170-178.

4. Berry RB, Chediak A, Brown LK, Finder J, Gozal D, Iber C, et al. Best clinical practices for the sleep center adjustment of noninvasive positive pressure ventilation (NPPV) in stable chronic alveolar hypoventilation syndromes. J Clin Sleep Med 2010;6(5):491-509.

5. Jounieaux V, Aubert G, Dury M, Delguste P, Rodenstein DO. Effects of nasal positive-pressure hyperventilation on the glottis in normal awake subjects. J Appl Physiol 1995;79(1):176-185.

6. Jounieaux V, Aubert G, Dury M, Delguste P, Rodenstein DO. Effects of nasal positive-pressure hyperventilation on the glottis in normal sleeping subjects. J Appl Physiol 1995;79(1):186-193.

7. Janssens JP, Borel JC, Pépin JL, SomnoNIV Group. Nocturnal monitoring of home non-invasive ventilation: the contribution of simple tools such as pulse oximetry, capnography, built-in ventilator software and autonomic markers of sleep fragmentation. Thorax 2011; 66(5):438-445.
8. Schönhofer B, Sortor-Leger S. Equipment needs for noninvasive mechanical ventilation. Eur Respir J 2002;20(4):1029-1036.

9. Massie CA, Hart RW. Clinical outcomes related to interface type in patients with obstructive sleep apnea/hypopnea syndrome who are using continuous positive airway pressure. Chest 2003;123(4):1112-1118.

10. Mortimore IL, Whittle AT, Douglas NJ. Comparison of nose and face mask CPAP therapy for sleep apnoea. Thorax 1998;53(4):290-292.

11. Teo M, Amis T, Lee S, Falland K, Lambert S, Wheatley J. Equivalence of nasal and oronasal masks during initial CPAP titration for obstructive sleep apnea syndrome. Sleep 2011;34(7):951-955.

12. Schorr F, Genta PR, Gregório MG, Danzi-Soares NJ, Lorenzi-Filho G. Continuous positive airway pressure delivered by oronasal mask may not be effective for obstructive sleep apnoea. Eur Respir J 2012;40(2):503-505.

13. Vrijsen B, Buyse B, Belge C, Testelmans D. Upper airway obstruction during noninvasive ventilation induced by the use of an oronasal mask. J Clin Sleep Med 2014;10(9):1033-1035.

14. Clinical indications for noninvasive positive pressure ventilation in chronic respiratory failure due to restrictive lung disease, COPD, and nocturnal hypoventilation: a consensus conference report. Chest 1999; 116(2):521-534.

15. Jounieaux V, Parreira VF, Aubert G, Dury M, Delguste P, Rodenstein DO. Effects of hypocapnic hyperventilation on the response to hypoxia in normal subjects receiving intermittent positive-pressure ventilation. Chest 2002;121(4):1141-1148.

16. Bourke SC, Bullock RE, Williams TL, Shaw PJ, Gibson GJ. Noninvasive ventilation in ALS: indications and effect on quality of life. Neurology 2003;61(2):171-177.

17. Farrero E, Prats E, Povedano M, Martinez-Matos JA, Manresa F, Escarrabill J. Survival in amyotrophic lateral sclerosis with home mechanical ventilation: the impact of systematic respiratory assessment and bulbar involvement. Chest 2005;127(6):2132-2138.

18. Georges M, Attali V, Golmard JL, Morélot-Panzini C, Crevier-Buchman L, Collet JM, et al. Reduced survival in patients with ALS with upper airway obstructive events on non-invasive ventilation. J Neurol Neurosurg Psychiatry 2016;87(10):1045-1050.

19. Ito K, Chitose H, Kobayashi A. [Upper airway obstruction caused by a floppy epiglottis-report of two cases of amyotrophic lateral sclerosis (ALS)]. Nihon Jibiinkoka Gakkai Kaiho 2009;112(9):660-664.

20. Sanches I, Martins V, dos Santos JM. Obstructive sleep apnea refractory to treatment due to floppy epiglottis. Arch Bronconeumol 2015;51(2):94.

21. Andersen T, Sandnes A, Hilland M, Halvorsen T, Fondenes O, Heimdal $\mathrm{JH}$, et al. Laryngeal response patterns to mechanical insufflationexsufflation in healthy subjects. Am J Phys Med Rehabil 2013; 92(10):920-929.

22. Kwok H, McCormack J, Cece R, Houtchens J, Hill NS. Controlled trial of oronasal versus nasal mask ventilation in the treatment of acute respiratory failure. Crit Care Med 2003;31(2):468-473.

23. Maurer JT, Stuck BA, Hein G, Hörmann K. Videoendoscopic assessment of uncommon sites of upper airway obstruction during sleep. Sleep Breath 2000;4(3):131-136.

24. Kaminska M, Montpetit A, Mathieu A, Jobin V, Morisson F, Mayer P. Higher effective oronasal versus nasal continuous positive airway pressure in obstructive sleep apnea: effect of mandibular stabilization. Can Respir J 2014;21(4):234-238.

25. Cavaliere M, Russo F, Iemma M. Awake versus drug-induced sleep endoscopy: evaluation of airway obstruction in obstructive sleep apnea/hypopnoea syndrome. Laryngoscope 2013;123(9):2315-2318.

26. Georges M, Adler D, Contal O, Espa F, Perrig S, Pepin JL, et al. Reliability of apnea-hypopnea index measured by a home bi-level pressure support ventilator versus a polysomnographic assessment. Respir Care 2015;60(7):1051-1056. 